\title{
LEVELS OF CONSCIOUS AND UNCONSCIOUS ANTICIPATORY BEHAVIOUR FOR ARTIFICIAL CREATURES
}

\author{
Pavel Nahodil \\ Department of Cybernetics \\ Czech Technical University in Prague \\ Technická 2, 16627 Prague, Czech Republic \\ E-mail: nahodil@,fel.cvut.cz
}

\section{KEYWORDS}

Anticipation, Simulation, Agents, MAS, Artificial Life, Creatures, Behaviour, AI, Intelligent Robotics.

\begin{abstract}
Recently, anticipation and anticipatory learning systems have gained increasing attention in the field. The interest of researchers in anticipation did not started over night. Anticipation observed in the animals combined with the multi-agent systems and artificial life gave birth to the anticipatory behaviour. This is broad multidisciplinary topic, but there are little thoughts on relation of anticipation with the reactive behaviour, the similarities and where the boundary is. Reactive behaviour is still considered as the exact opposite for the anticipatory one. It was shown by us that reactive and anticipatory behaviour can be combined. Designed multi-level anticipatory behaviour approach is based on the current understanding of anticipation from both the artificial intelligence and the biology point of view. Original thought is that we use not one but multiple levels of unconscious and conscious anticipation in a creature design. The topic is quite comprehensive and is out of scope of a single article to describe all 8 levels of the 8-factor anticipation framework design. The aim is not to extensively present all the achieved results but to demonstrate the thinking behind. Primary industrial application of this approach is intelligent robotics.
\end{abstract}

\section{INTRODUCTION}

Man desire for copying the creations of Mother Nature reaches deep into the history. In the second half of the 20th century robotics as a science branch started to advance rapidly. Robots started to be smaller, smarter and autonomous. Scientist all over the world focused on various areas or robot control starting from the space orientation, navigation through recognition, adaptation up to the cooperation in teams and behaviour.

The autonomous operation of robot in real environment meets many challenges. The control architecture must give the robot ability to react timely with respect to local disturbances and uncertainties and adapt to more persistent changes in environmental conditions and task requirements. The inherent problem of this area of research is that considerable work effort is required to equip robots with adequate means for sensing (sensors) and actuation (effectors). Recognition and transformation of data in noisy and voluminous environment poses an obstacle in the robot design. Therefore, to study control architecture, for us the most interesting part, the research moved from real environments to virtual environments. The term "agent" replaced the term "robot". The research mainly of behaviour no longer needs a physical robot; the virtual representation of the robot can provide the same level of embolism as a real one. For such virtual robots in analogy with the multi-agent systems (MAS) the term "agent" stated to be used. By this two fundamentally different approaches were joined by the selection of common name "agent". MAS originally used the topdown approach, focused on planning, problem solving which we can consider as a high-level function of some animals and humans (Huber 2010). On the contrary the bottom-up approach used in the robotics and originally by nature in the simple organisms and animals is more focus on reactions to the stimuli. This approach uses emergence as a tool for creating more complex and complicated behaviour by chaining the basic reaction together. By joining these two approaches together with meaningful trade off between theirs pros and cons proved to be an interesting approach. On the top of this, the interest turned in last decade to the ability to predict future changes and preparation for them, term anticipation started to be used for this topic.

The presented research builds upon knowledge from several scientific fields. Ethology / biology contributed by the various examples and experiments with animals focused on the explanation of the behaviour and the mechanism that produces and selects the behaviour not only as a reaction to the current state but also as a reaction to estimated future states and preparation for them. Control engineering provided a framework for formalizing systems, describing, controlling and modelling them and also estimating their future states. Classical Artificial Intelligence (AI) offered the high deliberative functions like learning, planning, reasoning and others.

Described approach to anticipation is inspired by works of several researchers such as Dubois, Butz, Martinho and Stolzman. The novelty of our work is in two main ideas. The first idea is bringing the distinction between conscious and unconscious anticipation. The second one is in the classification of different types of anticipation. 


\section{ARTIFICIAL LIFE APPROACH}

The term Artificial Life (ALife) literally means "life made by humans rather than by nature". ALife approach (biological model) of implementation of intelligent behaviour is inspired mostly by nature phenomena, instead of classical artificial intelligence (rational approach) which is more concerned about logic, rationality and just partially on algorithms inspired by nature. Another significant difference between AI and ALife approach is in the object of interest. Artificial intelligence is focused on complex thinking tasks, such as for example chess playing, text understanding, disease diagnostic etc. ALife on the other hand focused on the very basic elements of natural behaviour with stress on survival in changing environment.

The most of existing ALife approaches are based on algorithms which enables autonomous robots, as artificially created creatures, to evolve and adapt. These algorithms are inspired almost exclusively by biological systems. They have contributed the development of a large number of artificial intelligence techniques such as neural network architectures, genetic and evolutionary programming, robotic and multi-agent systems and others. Each biological system brings a large amount of evolutionary baggage unnecessary to support intelligent behaviour, but focusing not on content but on principles, the study of animal behaviour can provide a lot of models that can be successfully implemented within artificial (robotic or agent) system. Due to the inherent complexity of these systems a multi-level analysis approach supported with a lot of experiments and simulations is required. The common technique is to pick only one aspect or phenomena convert it to the algorithm and test it functionality in simulation. Animals, in contrast to the majority of agent applications, in which agents are highly specialized in terms of behaviour, deployment environment, learning capabilities etc., incorporate broad set of behaviours and high level of adaptability, mobility, social capabilities, proactivity, reactivity, and are employing various learning methods in one system. It is still a challenging task to mimic all of this in artificial creature including the wide set of behaviours such as predator avoidance, nesting, fighting, eating, exploring, sleeping, reproduction and others. With computational power of nowadays computers we have reached the point when we can imitate Mother Nature. ALife gives us chance to test our comprehension of intelligence, adaptation for living conditions and evolution (Kohout and Nahodil 2010).

The philosophy of classical MAS has been reversed in Artificial Life. ALife draws inspiration from many science disciplines such as biology, ethology, sociology, psychology, mathematics (grammars) and physics. ALife is often being connected with the phenomena of emergence. By mutual local cooperation of primitives a new phenomenon on global level arises. Principle of superposition does not work here, the sum of the parts is better than the whole (nonlinear behaviour of elemental primitives). This is called emergence and is achieved without any central control. Tools for evolution towards more complex and more perfect structures are selfreproduction, mutation and selection.

\section{ANTICIPATION AND EMOTIONS}

Basic definition of anticipatory systems was published in 1985 by biocyberneticist Robert Rosen in his book Anticipatory systems. He defined an anticipatory system as follows: "A system containing a predictive model of itself and/or its environment, which allows it to change state at an instant in accord with the model's predictions pertaining to a latter instant". Rosen in his book was inspired by his observation of live organisms, namely the ones with higher intelligence. Especially by their ability to predict the future and make adaptations based on them. This ability of live beings was already discovered before. Rosen however utilizing this knowledge, created a theory which was abstracted for various systems. All beings of nature use anticipation whether they are aware of it or not.

What is not that obvious is that anticipation is not matter of a single mechanism or a control block in a living organism. Anticipation happens on many different levels even in the very simple creatures. The works studying anticipation in the past overlooked this fact, focusing on the anticipatory principle or mechanism only. And that exactly allowed us to use not one but several levels of anticipation in a creature design and to observe the results. Our approach is to our knowledge one of the first that looks at the conscious and unconscious part of anticipation separately.

As an example showing different level of anticipation, to explain our postulate above about different levels, I can use something very common a tennis player. While playing the opponent a player is trying to estimate (based on measured observations) the opponent's action and also the result of his action (trajectory of the ball). This estimate is based on the "model" the player has. This model contains among other the human body physiology, the laws of gravity and physics. These are not expressed as equations but as patterns and rules for reaction. These patterns and responses can be improved by training. The prediction of future state plays important role in closing the loop of regulation (control) namely. The prediction influences the decision about current state anticipation and the behaviour influenced by the future state anticipatory behaviour. This clearly suggests that anticipation is not just another function of the organism; it is built in and applied on different levels (Huber 2010).

The definition of what exactly is an emotion in an anticipatory system is not a trivial matter. Consider, for instance, surprise, a central concept in anticipatory systems, as non-anticipatory systems cannot be surprised. This is clear, surprises come from expectations, and if there is no expectation there cannot be a surprise. Or better the surprise can still happen but it will not be perceived like one by the non-anticipatory system. Although all anticipatory systems deal with 
surprise in one way or another (ignore it, evaluate the reinforcement, etc...) not all systems consider it to be truly affective state. It is important to understand the possible relations between emotion and anticipation, to disentangle very different structural and psychological relationships between the two concepts. There are three different relationships between emotion and anticipation according to work of Carlos Martinho (Martinho 2007):

- Emotions eliciting an anticipatory behaviour (as the tendency to act),

- Emotions resulting from eliciting or confronting anticipatory representations (where emotions can be seen as such a representation),

- Anticipating future emotions (in the sense that there is a special pathway for emotions when compared to other representation pathways).

Emotions are important part of the behaviour of each being but it is really hard to provide an exact definition. Mostly due by its ubiquity, emotion can be approached in many ways in psychology and other related disciplines. It would be closed-minded not to utilize the knowledge about emotions and how they influence behaviour in artificial creature design. I already mentioned in the introduction that ALife is an interdisciplinary science. Theories from various disciplines such as (biology, sociology, anthropology, neurophysiology, cultural studies and philosophy) show how emotions are not only an inter-individual matter, that goes far as language and culture, and have a good heuristic value. Beyond emotion, no other aspect of human condition is at-ease in adopting a multidisciplinary and plural approach. Any other approach would be too simplistic, and would not make justice to the fascinating field of emotion (Dubois 2004).

It may seem that these two examples bear little resemblance however they have something in common. In both the prediction of future state plays important role in closing the loop of regulation (control) namely. The prediction influences the decision about current state anticipation and the behaviour influenced by the future state anticipatory behaviour. This clearly suggests that anticipation is not just another function of the organism; it is built in and applied on different levels.

Main goal of my research group is to implement anticipation in the agent architecture. So initially we focused on revision of the current theories and then we tried to formulate own theory. We have received a proof that anticipation, implemented even on these multiple levels can be beneficial with much better results. The main reson for this is, that anticipated values and states provide additional input to the decision making process, we like to say it brings a new dimension. This enables for various improvements of the behaviour patterns.

The anticipation can be applied in variety of areas, because it goes beyond the future state estimation. It provides this as an additional input to decision or regulation mechanism. Primary industrial application of our approach is intelligent robotics and humanoids.

\section{MULTI-LEVEL ANTICIPATION STRUCTURE}

It is obvious that there seems to be several types of anticipation as shown above. Even though we embrace the categorization of Butz Anticipation (Butz et al 2003), we were not entirely satisfied with it. As shown below we struggle to categorize his 4 basic types from the point of control of the creature's will. We solved this by applying each category on conscious and unconscious level, creating thus 8 types of Anticipation which we call Multi-Level Anticipation or 8-Factor Anticipation - $8 F A$ (Kohout 2011). This is an original and novel contribution to the Theory of Anticipation. We came up with and will father develop and research. All the levels are schematically shown on Figure 1.

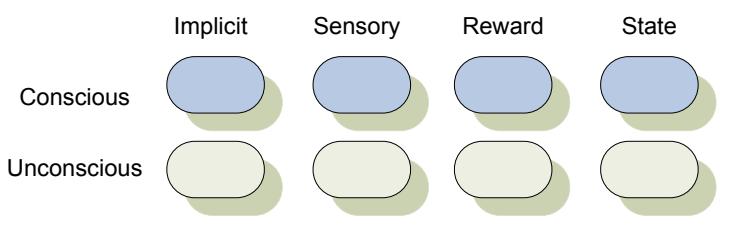

Figure 1: The 8-Factor Anticipation

There are these 8 sorts of Anticipation: Unconscious Implicit, Conscious Implicit, Unconscious Sensory, Conscious Sensory, Unconscious Reward, Conscious Reward, Unconscious State, and Conscious State.

We can say that the complexity grows in the picture from the left to the right and from the bottom to the top. Generaly an architecture spans through multiple levels. This means that the current works cannot be categorized by just one level, rather by a collection of levels they cover. Firstly, I will describe our understanding of all levels, then I will present an explanatory experiment we conducted to further demonstrate the ideas. To compare the work with other and statistically evaluate, we would need to encompass more than these two levels.

Anticipation is a characteristic of the living, but not a specific action or activity. We do not undertake anticipation. It is not a specific task. Anticipation is the result of a variety of processes. We are in anticipation. These are basic ideas that should be understood to successfully explore the broad field of anticipatory behaviour. As an outcome, anticipation is expressed through consequences like increased performance (an anticipated tennis serve is returned, danger such as a passing car is avoided, an opportunity in the stock market is used to advantage). Anticipatory processes are defined in contrast to reaction. The living learns, but some of its anticipatory characteristics are inherited. Better yet, they are implicit in the condition of being alive, or implicit in survival. Adventure, as an inclination, is telling more of survival than of curiosity unless curiosity itself is related to survival.

This paper greatly contributes to the understanding of differences between anticipation and other processes like guessing, expecting, forecasting, planning and predicting. It is vital to understand these differences in order to further investigate the possibilities of anticipation. 


\section{Unconscious Implicit Anticipation}

Under Unconscious Implicit Anticipation, we understand the behaviour that was imprinted in the creature by Nature (evolution) or by a creator (in artificial case) and that is not voluntary. Reactions and reactive behaviours itself is not anticipatory. Reaction is very often used and wrong understood as an opposite to anticipation (Kohout and Nahodil 2010). A possible area of involving of Implicit and Unconscious Anticipation is in inner agent processes that try to imitate the creature physiology, energy management, and internal processes. The inner process can either directly or indirectly influence the decision process. If it influences the decision indirectly than it needs some regulation system. This regulation system is hardcoded within the organism and is not consciously controlled hence it can be classified as Implicit Unconscious Anticipation.

We described here the first and the most basic anticipatory factor from our 8-Factor Anticipation Framework (8FA) and also suggested and discussed improvement to the implementation that might under certain circumstances increase the efficiency of the artificial creature.

\section{Conscious Implicit Anticipation}

The combination may seem illogical because implicit anticipation is something imprinted in the creature by design. How can this be consciously controlled?

Here still everything depends on the design but the results are available to the higher levels and also higher levels data such as desired state (converted to the desired value in the current step) are available as inputs. We described the second factor of our 8-Factor Anticipation Framework (8FA) here. This level leverages so called emotivector, introduced by Carlos Martinho in his thesis (Martinho 2007). Three modifications of the emotivector were suggested. The first one being anticipation of an input second being the rateability and last usage of meta-estimators with a priority queue. Only the second was broad tested in experiments and results provided as the other two do not need experimental confirmation.

\section{Unconscious Sensory Anticipation}

Moving on to the Sensory Anticipation on the unconscious level concludes all the sensory input gathering, pre-processing and data filtering. Basically here we can meet all the functions that cannot be voluntarily influenced. This information is collected, processed, stored or disregarded based on the attention and other factors. We can simulate the situation where the input magnitude is so huge that it cannot be processed all by the conscious processes. The key role here plays the estimation approaches chosen to provide the estimate. The function that we sometimes require to get closer to the animal world is filtering the information in order to reduce the information value - so more less informative data can be processed at the same time. - In this part we described the approach to estimate the next value on the level of sensor. At this level there is no context added, the next values are based on the historic values and the selected criteria (minimum squares, maximum likelihood) are computed. Please note that prediction of the value is in our design just a mechanism used to produce estimate that is processed further by other levels to generate the behaviour including emotions.

\section{Conscious Sensory Anticipation}

We are on the Sensory Anticipation level now, so we have access to the sensor data and from the previous level even to the estimate of future data.

A dog hunting a rabbit does not need to sense the hare continuously. If the hare, for example, disappears behind a bush - the dog predicts the future location of the hare by anticipating where it is going to turn up next and continues its hunt in this direction. This behaviour described below needs little bit more than a pure sensory input. It requires recognition of the objects (rabbit, bush) and making projections of a sensory data that cannot be directly measured at the moment by sensors. Also some knowledge about the rabbit and the environment has come into play. This means that we would need some already stored data to be recalled from the memory and associated with the recognized objects. Several necessary modifications to the reference algorithm were required to adapt it to our environment. We suggested another modification using the estimators to aid the probability distribution. The main point of this contribution is that this is a first example how the levels can support each other and work together. This is one of the key aims for our work.

\section{Unconscious Reward Anticipation}

The reward or better said reinforcement to include also punishment is a powerful way to learning.

There are countless approaches, theories and their modifications using reinforcement to create adaptive artificial creatures. Reinforcements together with the expectations (anticipation) also serve to generate emotions. Now we are at the level of unconsciousness which means that information should be processed in quick and simple manner to have a decision ready in case the conscious part is still processing the data but the action is needed at the moment. So the first requirement is a fast and easy computing. The second consideration we need to do is about emotions. If we want our creature to experience emotions we need to generate them somehow. In our opinion emotions originate in the unconscious level but they are recognized by the consciousness and can be there partially controlled.

We focused mainly on emotional state generation here. We have discovered several weaknesses of the emotivector approach and extended the theory to cover more complex simulation. We could introduce an emotion cube, which addresses the emotion 
superposition in a complex environment where more than one emotion is generated from the sensory inputs.

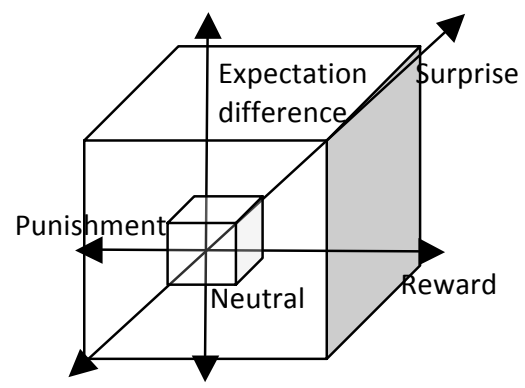

Figure 2: The Emotion Cube

\section{Conscious Reward Anticipation}

At this level we are finally reaching full capabilities of the current architectures. At this stage we are looking for framework that works with reward, and is able of working with the observations and gained knowledge including creating, modifying and deletion. We need to keep in mind that these might serve for other conscious levels to work on and hence they need to be compatible or abstract enough so all levels can understand them. This also means that the system should be open enough in terms of inputs it requires and outputs it provides so we can easily integrate it in the complex architecture.

We have argued what algorithm is right to use within our framework. Since the selected algorithm is ACS which has countless tested modifications. We focused on the integration of the algorithm with the rest of the architecture. The reason for this is, that if the first 6 levels are implemented it created already a complex artificial creature with anticipatory behaviour. This means that a subset of our 8-factor anticipatory design for consistency called 6-factor anticipatory design meets the goals of our work fully. As shown in the text, the first six levels conclude implicit, sensory and reward anticipation. These gives the artificial life agent the necessary prerequisites to be able to sense and learn in the environment. The other two levels gives the agent additional reasoning and deliberative capabilities.

\section{Unconscious State Anticipation}

This is the last of unconscious levels at the same time the most sophisticated and complex one from the unconscious levels. It has a similar problem to the Conscious Implicit Anticipation. The combination itself seems at the first sight confusing. However it is important part of the architecture and has its meaning. One example is the internal state of the creature. It is monitored through internal sensors, it is regulated and working without external actions needed but some unusual states should be reported to the conscious levels. The motivation was taken from the nature as always. So anticipatory monitors using the information about current actions and observed internal values behaviour would help to optimise the system and bring it again from reactive to anticipatory.

At this level we are creating additional information about the environment and the agent internal state that can be useful in complex scenarios. For example, learning the path (for example in a maze) could be used when a goal to reach the end of the maze is presented. This information can or cannot be utilized which means that it complicates the architecture which means that it will be slower and might lead to sub-optimal solutions compared with the 6-factor architecture. However the benefit is in additional reasoning capability and more "life-like" behaviour that we strive to achieve.

\section{Conscious State Anticipation}

The most complex and therefore the most interesting factor of 8FA is Conscious State Anticipation.

Basically all the classical AI approaches can find their place here starting from state space searching through planning up to the reasoning about others and self. These tasks typically require more time to process or let's say spare time. This can be imagined as a state of the agent where there is no urgent internal need to pursue any goal (food, sleep, etc...). Also the agent external goals are satisfied. In that case the agent can select the action to be to build, review, updated or evaluate the model of its own state or of others.

This level is very general and basically since it is a last level it can be used as an abstraction for any mechanism that was missed in the previous levels and is in the particular design needed. In general a long list of approaches can be taken (from which some were described in this work). It can have the shape of Kalman filter, temporal logic, state space search, planning, etc...

\section{EXPERIMENTS AND OBTAINED RESULTS}

Simulations in the virtual environment is the only way (for ALife in most cases) how to test theories and compare effectiveness of results with others. We have conducted our experiments in the REPAST simulation tool (REPAST 2010). Because our angle of view is novel and might be hard to grasp, I demonstrate this on the simplest possible examples in order to focus solely on the main ideas of described work.

In the experiements I will skip the first two levels, as they have been already explained in published works (Kohout and Nahodil 2010), (Kohout 2010), and I will focus on the sensory, reward and state levels in this article. The examples of the conscious sensory level are showing the object persistence.

\section{Conscious Sensory and Unconscious Reward Anticipation}

This example is to show not only the principles of object persistence belonging to the conscious sensory anticipation level. It is also presented in conjuction with eotion generation of the unconscious reward level and thus demonstrating that the different levels we described 
are not perceived as a standalone entities but as an interral parts of the 8-factor architecture.

For this experiment we have an agent ("wolf") observing another agent ("pig") and estimating it's position after it disappears behind the wall (this is the conscious sensory anticipation). Once the observed agent is visible again and the observing agent can verify his expectation an emotion can be generated, also the intensity of the emotion is evaluated and the final emotional state generated. For this purpose the behaviour of the observing agent was modified and once it is hidden, it can decide to turn back an continue it's motion counterclockwise, which will lead to surprise and also to difference between anticipated and real state.
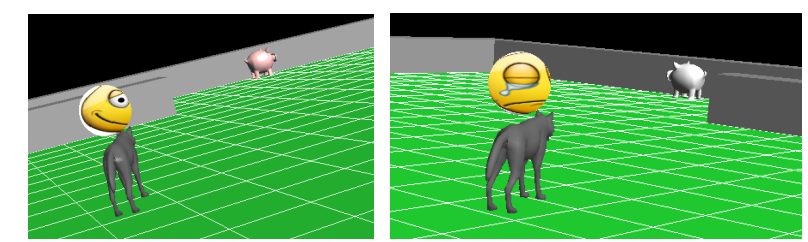

Figure 3: Emotion Generation - confirmed expectation leading to joy (left), unconfirmed expectation leading to suffering (right)

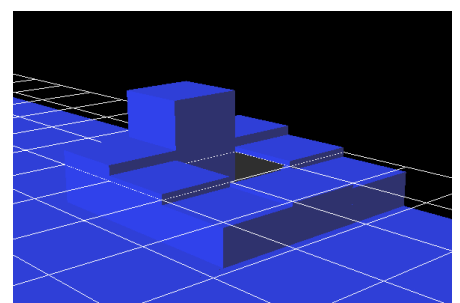

Figure 4: Probabilistic Occupancy Map - the visualization of the probability of object hidden behind obstacle

This experiment was used as a proof of concept and also to demonstrate several things. In isolation of levels it was proven that the comparison of expected and real values can be used to generate emotions. It was also shown that agent can successfully track an object even when hidden behind obstacle, based on the anticipation of the continuation of the movement. In the integration level, it was shown in this example that the object observation behind the wall can be one of the inputs into the generation of emotion.

\section{Conscious Reward and Unconscious State Anticipation}

The reason to demonstrate two levels together here is to show the tight integration of the levels with each other. The same implementation is used for these two levels. The Anticipatory Classifier System (ACS) was used to implement each level. Conscious reward anticipation is used fully consciously to achieve the goals and receive the reward. On the unconscious state level it is used for latent and thus unconscious learning process. The ACS serves as a method here how to capture the latent learned knowledge.
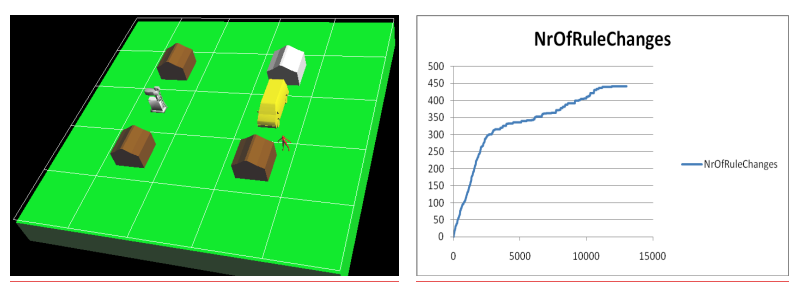

Figure 5: The Taxi Problem - visualization of the simulation (left), the convergence of the attempts to change the rule base (right)

In this scenario the main actor is a Taxi agent, shown as a yellow van. This agent's goal is to pickup Client agents shown as a red woman and takes them to their destination; the desired destination is shown as a white house, the rest of the houses are shown in brown. The Client agent is generated at random intervals. At a time there can be only one Client agent until the client is delivered to the final destination. The scenario contains also a filling station as the Taxi agent consumes energy by moving and transporting Client agents. Only one effector was defined for this scenario the agent can execute only one action at a time. The action can be to go up (0), go down (1), go left (2), to go right (3), to fill up the tank (4), to pickup the Customer (5), to drop off the Customer (6). Reward is presented to the agent in two situations. When the customer is successfully dropped off on the target destination $(\mathrm{r}=3)$, and when the car's tank is filled $(r=4)$.

For the latent learning we used similar setup to Stolzman (Stozlman 2000), and even he took the experiment from an ethology example of rat learning. In this scenario an agent is placed in a simple E shaped maze. The agent starts in the middle, and has a choice to go the left or to go the right. The end boxes have different color (black and white). The agent is allowed a free run in the maze pre-learning phase. After a certain period the agent is placed in the left side (black) and is presented with food (reward). Then the agent is again placed in the starting point. If the latent learning is correct the agent should be able to run straight to the left black box with anticipation of a reward there.

The goal of this paper is not only to explain the structure of anticipation algorithms and programs but also to present the useful applications of anticipation in our daily life. Anticipation can be used advantageously in such systems, which are very large and complex or the exact mathematical model would be too difficult or even impossible. In this respect we have started to work in some fields: "Smart Home", "Criminality Prevention", "Prediction of Terrorist Attack" and "Anticipated Collision Avoidance". Program Power Control simulates learned user's behaviour in a house and his influence of all devices in the house (system). Another part of program anticipates next state of system and tries to precede too high consumption of electricity by switching on and off some devices. The second goal is to increase the quality of user's life. Three methods of anticipation were designed and implemented: Markov chain, neural net and genetics programming. 
The other goal is to anticipate states with too large power take-off; it means to eliminate power spikes. There are defined these states:

\begin{tabular}{|l|l|}
\hline State & Total usage [\%] \\
\hline Low & $0-20$ \\
\hline Normal & $20-40$ \\
\hline High & $40-50$ \\
\hline Overload & $50-80$ \\
\hline Dangerous & $80-100$ \\
\hline
\end{tabular}

Program Power Control anticipates states Overload and Dangerous. If these states are anticipated, anticipatory control system tries to switch off some devices to receive the next state Low, Normal or High most highly. Program allows the simulation also without anticipation. The results are suitable for comparison with simulations with anticipation. We can see the quality of anticipation. Other possible anticipation applications are now not only in economic areas (exchange, inflation, etc.), but also in predicting earthquakes or volcanic activities and movement of dangerous hurricanes. Last but not least, the use of anticipation in the fight against global terrorism and protection of property and lives of individuals in general.

\section{CONCLUSION}

In this article, I presented the latest results of the longterm research achieved in my working group on the field of new artificial live approaches. Novel design of multi-level anticipation structure - 8FA is among them undoubtedly, too. Our doctoral student Karel Kohout, whom I have been a $\mathrm{PhD}$ supervisor, had focused on the topic of the Unconscious and Conscious Anticipation from "8 - Factor Anticipation Framework" (8FA), designed by him. In his thesis (Kohout 2011), it was shown, that the reactive behaviour employs anticipation inherited by the creator (nature, designer).

The main messages and contributions of our research are to draw an attention to anticipation as a mechanism involved almost in every aspect of the artificial creature behaviour. Anticipation is no longer an isolated term, it is an essential part of the artificial creature design (Kohout 2010). The interesting topic of consciousness and control in the background of anticipation is drawing more attention recently, as this point of view was noted by several researches. The fact that consciousness was a main topic of a recent conference CASYS 2010 shows that the interest on this topic among the anticipation community is growing. The complexity of anticipation and that simple categorizations are not able to comprise it was noted in recent works as well. The other intention of this article is to draw attention to an approach 8FA (described above) that I consider novel in the field of anticipation (Kohout and Nahodil 2010).

The aim was not to extensively present all the results but to demonstrate the thinking behind. I wanted to show that even in the basics are possibilities which can be further extended in the higher levels. I argue that even this simple overview shows a new way of thinking about anticipation not used before. The main conclusion to be made from this article is that consciousness plays important role and that anticipation is not matter of just one mechanism.

Author believe that this paper will be the good basis for further useful applications of anticipation.

\section{ACKNOWLEDGMENT}

This research has been funded by the Dept. of Cybernetics, Faculty of Electrical Engineering, Czech Technical University in Prague and Centre for Applied Cybernetics under Project 1M0567.

\section{AUTHOR BIOGRAPHIES}

PAVEL NAHODIL graduated with highest honors in Computer Science. He obtained his scientific degree Ph.D. from the Czech Technical University in Prague in 1980. Since 1986 he has been a Professor of Technical Cybernetics at the Dept. of Cybernetics, CTU in Prague, Czech Republic. His present professional interest includes artificial intelligence, multi-agent systems, intelligent robotics (control systems of humanoids) and artificial life approaches in general. He is (co-) author of several books, university lecture notes, hundreds of scientific papers and some collection of scientific studies. He is many international conferences organizer + IPC reviewer and a member of many Editorial Boards.

\section{REFERENCES}

Butz, M. V et al. 2003. "Anticipatory Behavior” In: Adaptive Learning Systems: Foundations, Theories, and Systems, Springer Verlag, Berlin, 303 pp.

Dubois, D. 2004 "Mathematical Foundation of Discrete and Functional Systems with Strong and Weak Anticipation." In: Anticipatory Behaviour in Adaptive Learning Systems. LNCS 2684, Heidelberg: Springer Verlag. pp. 110 - 132.

Huber, M. and Glasauer, S. 2010. "When to assist? Modelling human behaviour for hybrid assembly systems". In Proc. ISR 2010 - 21st Intern.Symposium on Robotics. Munich: VDE Verlag, pp. 321 - 327.

Kohout, K. 2010. "Conscious and Unconscious Implicit Anticipation." DAAAM International Scientific Book 2010, DAAAM International Vienna Verlag, Scientific Book, Vol .9, Chap. 46, pp. 525-534.

Kohout, K. and Nahodil, P. 2010. "Reactively and Anticipatory Behaving Agents for Artificial Life Simulations.” In: CASYS 2010 - Computing Anticipatory Systems. Editor Dubois D. AIP, 2010. pp. 302 - 309.

Kohout, K. 2011. "Anticipatory Behaviour for Alife Domain: Multi-level Anticipation Structure". PhD Thesis. Prague: Dept. of Cybernetics, Czech TU in Prague, 149 pp.

Martinho, C. 2007. "Emotivector: Affective Anticipatory Mechanism for Synthetic Characters." PhD Thesis. Lisbon: Instituto Superior Técnico, TU in Lisbon, $132 \mathrm{pp}$.

REPAST [online]. Last revision 2010. [cit. 2011-04-10]. http://repast.sourceforge.net/download.html

STOLZMANN, W. 2000. "An Introduction to Anticipatory Classifier Systems”. In: LNCS 1813 - Learning Classifier Systems. Springer Verlag, Berlin, pp. $175-194$. 